\title{
DE MACROCONTINGÊNCIAS A METACONTINGÊNCIAS NO JOGO DILEMA DOS COMUNS
}

\section{FROM MACROCONTINGENCIES TO METACONTINGENCIES IN THE DILEMMA OF COMMONS}

\author{
ELAYNE ESMERALDO NOGUEIRA ${ }^{1}$ \\ LaERCIa ABREu VAsconcelos²
}

UNIVERSIDADE DE BRASÍLIA, BRASIL

\section{RESUMO}

O objetivo deste estudo foi realizar um análogo experimental de uma macrocontingência utilizando-se do Dilema dos Comuns. Três participantes recebiam 100 peixes e retiravam 2, 4 ou 6 a cada tentativa. Havia um reajuste contingente ao número retirado e o restante de peixes era informado. Na linha de Base as escolhas eram sigilosas, em B1 eram divulgadas e em B2 os jogadores conversavam. Foram 3 grupos experimentais com alteração da ordem das condições experimentais e um grupo controle. Houve extinção dos recursos no grupo controle e nas primeiras linhas de base, que foram analisadas como uma macrocontingência. Ocorreu o aumento dos recursos nas condições B com o entrelaçamento das escolhas dos participantes, analisando-se como um análogo experimental de uma metacontingência.

Palavras-chave: ambiente virtual, reforço, discriminação simples, reversão de discriminação, crianças.

The aim of this study was to conduct an experimental analogue of a macrocontingency using the Dilemma of Common. Three participants were given 100 fish and withdrew 2, 4 or 6 at each attempt. There was a contingent adjustment about the number removed and the number of remaining fish was informed. In the Base line, the choices were classified, in B1 they were disclosed, and in B2 players talked to each other. There were 3 groups with changes in the order of the experimental conditions, and a control group. There was extinction of resources in the control group and in the first baselines, which were analyzed as a macrocontingency. There was an increase of resources in conditions B with interlacing of participants' choices, analyzed as an experimental analogue of a metacontingency.

Keywords: Macrocontingency, metacontingency, Dilemma of the Common

1. Analista Judiciária - especialidade psicologia - do Tribunal de Justiça da Paraíba - laynesmeraldo@ gmail.com

2. Professora de Pós-Graduação em Ciências do Comportamento da Universidade de Brasília - laercia@unb.br 
Skinner (1953) relaciona a análise do ambiente social ao conceito de comportamentos de pessoas em grupo, afirmando que "o efeito de um ambiente social no comportamento pode ser inferido ponto por ponto de uma análise daquele ambiente" (p. 325). Nesse sentido, o autor defende que a análise do comportamento social deve ser baseada nos mesmos princípios da análise individual do comportamento, em que um comportamento possui uma função em seu contexto de origem. No comportamento social, o comportamento de um organismo se torna parte do ambiente que controla o comportamento de outro organismo e, em um grupo, o controle social entre os membros pode ter um efeito poderoso nos comportamentos dos indivíduos desse grupo. Skinner (1953) ressaltou que quando duas ou mais pessoas manipulam variáveis de um grupo, isso tem um efeito comum sobre seu comportamento.

Nesse sentido, a análise do comportamento social deve ser baseada nos princípios da análise do comportamento individual, em que um comportamento possui uma função em seu contexto de origem. No comportamento social os organismos se tornam contexto para outros organismos. Em 1981, Skinner estabelece o surgimento do comportamento verbal como o elo entre o nível ontogenético e cultural, designando esse último nível como responsável pela seleção de práticas culturais e sugerindo que por meio do comportamento verbal as pessoas se tornariam mais cooperativas diante de empreendimentos comuns. Isso possibilitaria a seleção de práticas culturais como solução de problemas para um grupo.

Glenn, (1986) propõe um novo conceito na análise do comportamento para estudar as práticas culturais. O conceito de metacontingências surgiu da análise das relações entre contingências operantes organizadas socialmente que possuem uma consequência a longo prazo, denominada de consequência cultural, responsável pela seleção dessas relações operantes socialmente organizadas. Em 1989, o comportamento verbal nas práticas culturais é destacado com a função de suporte das contingências não verbais, coordenando essas contingências, sendo o elo entre as contingências comportamentais entrelaçadas (Glenn, 1986;1989) e, ainda, sendo o responsável pela transmissão da prática a outros membros da comunidade verbal.

Posteriormente, o desenvolvimento dos conceitos analítico-comportamentais utilizados no estudo da evolução de práticas culturais, tais como macrocomportamentos, metacontingência, macrocontingência, linhagem culturo-comportamental e cunha cultural (cultural cusp) são apresentados em Glenn et al. (2016). Macrocomportamento é definido como "comportamento operante socialmente aprendido observado no repertório de vários membros de um sistema cultural." (p.18). Assim, "Macrocontingência é a relação entre 1) comportamento operante governado por contingências individuais e/ou CCEs governadas por metacontingências e 2) um efeito cumulativo de significância social." (p. 19).

$\mathrm{Na}$ macrocontingência os macrocomportamentos são selecionados em suas contingências específicas. $\mathrm{O}$ consumo de recursos renováveis na natureza ilustra tal conceito. A prática cultural seria um grande número de pessoas fazerem uso abusivo dos recursos de um determinado país, já que esses recursos são comuns a todos os seus cidadãos, enquanto o efeito cumulativo dessa prática seria a diminuição da quantidade desses recursos, ou, possivelmente, a extinção dos mesmos. Entretanto, apesar da diminuição desses recursos resultar do macrocomportamento de consumo dos recursos, não ocorre a seleção da prática cultural por tal efeito cumulativo, pois os comportamentos de um indivíduo não ocorrem em função dessa consequência, tendo cada comportamento sua consequência operante que o seleciona. Para que haja uma mudança nesses efeitos cumulativos seria necessário algo que promovesse a mudança dos comportamentos das várias pessoas envolvidas no processo (Glenn, 2004).

No entanto, Metacontingência contém pelo menos dois elementos: o culturante (CCEs + Produto Agregado) e um ambiente ou eventos selecionadores. O termo contingências comportamentais entrelaçadas (CCEs), já apontado por Skinner como um sistema entrelaçado de respostas $(1953,1957)$, foi definido por Glenn (1991), ressaltando o papel de ação e de ambiente para comportamentos de outros. Nesse sentido, um elemento que possui função de resposta em uma contingência $A$, pode adquirir função de ambiente social para outro comportamento em uma contingência B. Os culturantes podem ser eventualmente mantidos por essas consequências culturais na ausência de reforçamento direto para o comportamento do participante. Portanto, trata-se de uma relação funcional entre culturante e consequência cultural. E, vale ressaltar a possiblidade de expansão dos elementos constituintes de uma metacontingência para três ou quatro elementos (Glenn et al., 2016). As metacontingências são unidades de seleção cultural, conforme esquematizado na Figura 1.

Vários estudos experimentais têm sido realizados com o intuito do desenvolvimento do conceito de metacontingências: Amorim (2010); Brocal (2010); Gadelha (2010); Vieira (2010); Leite (2009); Cruz (2009); Bullerjhann, (2009); Caldas (2009); Martone (2008); Baia (2008); Pereira (2008) e Vichi (2004). É possível observar dois modelos experimentais até então desenvolvidos. O primeiro baseia-se na utilização de uma matriz com sinais positivos e negativos em que os participantes deveriam fazer suas escolhas. O segundo traz uma situação em que fileiras de caselas eram expostos aos participantes que deveriam escolher determinados números para preenchimento das caselas e havia pontos individuais e ganhos para o grupo a partir de suas escolhas. 


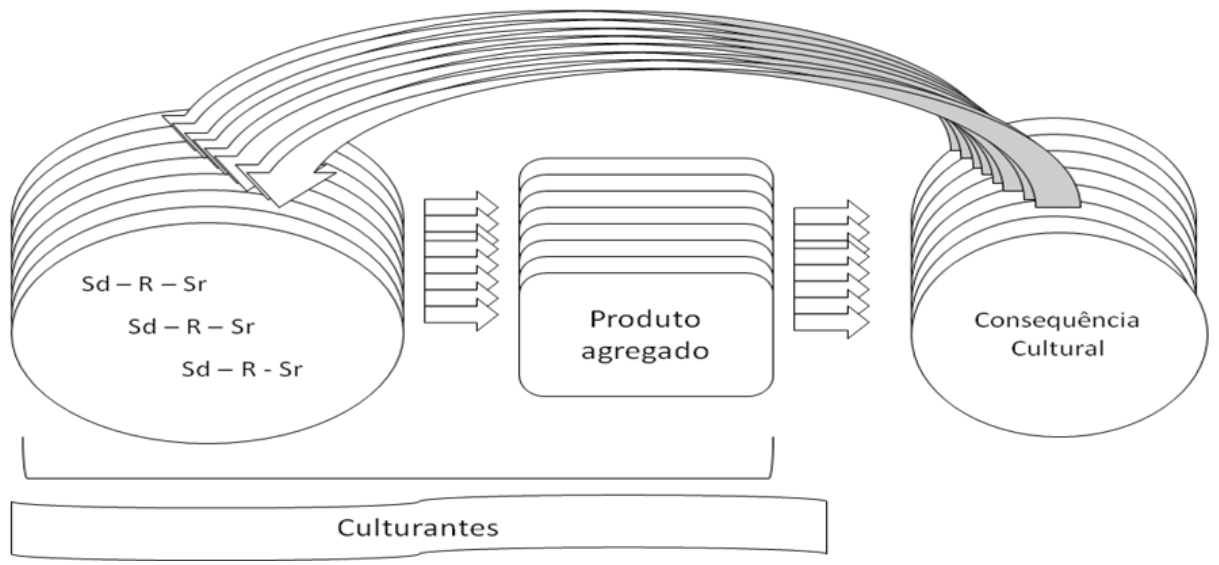

Figura 1. Esquema do conceito de metacontingências

Vichi (2004) utilizou uma matriz com sinais positivos e negativos em que os participantes deveriam fazer suas escolhas e inseriu uma consequência para o grupo, analisando se a mesma poderia selecionar as interações entre seus membros, mudando a consequência cultural diante do comportamento do grupo de cooperar e competir. Os resultados mostraram variações no comportamento do grupo a partir de modificações da consequência cultural. Martone (2008), a partir da replicação de Vichi (2004), utilizou-se também da transmissão cultural, presente no delineamento experimental de Baum e cols (2004), em que há trocas do participante mais antigo do grupo por um novo membro. Os resultados são consistentes com o estudo de Vichi (2004). Leite (2009) acrescenta variáveis de instrução com participantes confederados e história experimental prévia. Os resultados mostram que o grupo é sensível à consequência comum, havendo a seleção de interações entre os participantes e a transmissão cultural. Portanto, há influência recíproca sobre as escolhas dos participantes.

Pereira (2008), Caldas (2009), Bullerjan (2009), Amorim (2010); Brocal (2010); Gadelha (2010); Vieira (2010) utilizaram-se de uma situação em que era apresentado duas fileiras de caselas aos participantes contendo uma numeração de 0 a 9 na primeira fileira. Para ganho de pontos individuais os participantes deveriam apresentar determinados números nas caselas. $\mathrm{Na}$ contingência cultural, a soma dos números dos participantes era condição para ganho cultural. Em uma segunda condição do experimento, foi retirada a consequência cultural, denominando o experimento de extinção cultural, sendo avaliado o efeito também da retirada da consequência individual (Caldas, 2009; Brocal 2010). Bullerjan (2009) realizou o estudo com quatro participantes. Esse programa foi ainda utilizado para avaliação da seleção de diferentes produtos agregados, a presença de condições antecedentes em metacontingências e ainda a intermitência da consequência cultural. (Amorim, 2010; Gadelha, 2010; Vieira, 2010). Os resultados mostram que as interações entre os comportamentos dos participantes são sensíveis à consequência cultural, sendo possível a seleção de diferentes produtos agregados, observado o efeito de presença de condições antecedentes e a seleção de produtos agregados e CCEs com uma consequência cultural em reforçamento intermitente.

Baia (2008) e Cruz (2009) realizaram replicação de Baum e cols. (2004) com uso de escolha consensual entre os participantes e a transmissão cultural. No procedimento, havia a construção de uma tarefa pelo grupo e uma consequência cultural contingente a essa tarefa. Baia (2008) observou a modificação do comportamento dos membros do grupo a partir da consequência cultural. Cruz (2009) investigou a presença de regras cerimoniais e tecnológicas no controle do comportamento de escolha dos participantes. Os resultados mostram que as escolhas são sensíveis à consequência cultural, com destaque para as regras cerimoniais.

Borba e cols. (2014) realizaram um estudo que discute o autogerenciamento ético e respostas autocontroladas em situações de conflito entre consequências individuais e para um grupo. $\mathrm{O}$ arranjo experimental foi realizado com onze grupos de quatro estudantes cada que tinham que escolher entre linhas pares ou impares de uma matriz $8 \times 8$. No caso, foi utilizado um procedimento análogo ao de Vichi (2004), Martone (2008) e Leite (2009). Nesse experimento, a escolha pelas linhas impares produzia maior reforço individual e consequências aversivas atrasadas para o grupo, sendo denominadas de escolhas egoístas e impulsivas, enquanto a escolha de linhas pares produzia menor reforçamento individual, porem com consequências reforçadoras para o grupo a longo prazo, chamadas de escolhas éticas e autocontroladas. Na condição em que os participantes foram expostos a tarefa sozinhos, houve uma maior quantidade de escolhas egoístas. Na condição em que foram expostos a tarefa juntos e puderam ter acesso a escolhas dos outros e conversar entre si, houve uma maior quantidade de escolhas éticas e autocontroladas. Em outra condição, os participantes conversaram entre si, mas não tinham acesso direto à escolha do colega, mas mesmo assim houve uma maior taxa de escolhas autocontroladas. $\mathrm{Na}$ quarta condição, os participantes realizaram a tarefa juntos, porém não tinham acesso a escolha do outro e nem 
conversavam, tendo essa contingência produzido escolhas egoístas também. Assim, o presente estudo demonstrou que contingências de interação social e acesso a escolha dos outros aumentam a probabilidade de escolhas que produzam consequência de maior valor reforçador para o grupo, mesmo que isso signifique consequências de menor valor reforçador individual.

\section{Teoria dos Jogos - $\mathrm{O}$ fenômeno da tragédia dos comuns}

Jogos da matemática aplicada são instrumentos que auxiliam o desenvolvimento de pesquisas sobre práticas culturais, a partir da abordagem analíticocomportamental (e.g., Abele \& cols., 2005; Brechner, 1977; Costa, 2009; De Alencar, 2008; Jones \& Rachlin, 2009; Martichuski, 1991; Nogueira, 2009; Ortu \& cols., 2008; Yi \& Rachlin, 2009). A Teoria dos Jogos é uma área da matemática com estudos experimentais sobre o desenvolvimento de estratégias para as escolhas, comparando os percursos semelhantes entre os participantes. Os jogos podem também incluir situações sociais e dilemas presentes no ambiente natural (Dietz et al, 2001; Fiani, 2004; Lima, 2005).

A interface entre a matemática e estudos da psicologia tem sido observada na aplicação das teorias dos jogos em estudos experimentais (Abele \& Ehrhart 2005; Brechner, 1977; Costa, 2009; De Alencar, 2008; Jones \& Rachlin, 2009; Martichuski \& Bell, 1991; Nogueira, 2009; Ortu \& cols., 2008; Yi \& Rachlin, 2009). Yi e Rachlin (2009) aplicaram o jogo do Dilema dos Prisioneiros (PDG), investigando as relações entre o número de participantes e as estratégias adotadas pelos participantes. Cinco participantes jogavam com o computador que utilizava diferentes estratégias. Os participantes deveriam escolher entre X (competir) e Y (cooperar). Os resultados mostraram uma maior frequência de cooperação para os participantes na estratégia Tit for Tat (TFT) ${ }^{1}$.

Outros estudos (Costa, 2009; Nogueira, 2009; Ortu \& cols. 2008) uniram o conceito de metacontingência com a teoria de jogos. Uma consequência comum foi aplicada ao comportamento de todos os jogadores com o objetivo de selecionar determinados arranjos de CCEs, ou de estratégias adotadas pelos membros de um grupo jogando o PDG. (Ortu \& cols. 2008). Os resultados mostraram que a consequência comum a todo o grupo selecionou os comportamentos entrelaçados dos participantes no jogo. Costa (2009) replicou o estudo acrescentando grupos verbais (em que os participantes conversavam entre si) e não verbais (em que não havia qualquer diálogo entre os participantes), manipulando a interação verbal entre os participantes, investigando se seriam possíveis o entrelaçamento de comportamentos e a seleção dos mesmos em grupos em que os participantes não conversavam entre si, apenas sabiam a escolha do outro. $\mathrm{O}$ autor encontrou que a consequência cultural

\footnotetext{
${ }^{1}$ Essa estratégia se trata de repetir a escolha do outro participante na tentativa anterior.

Essa estratégia se trata de repetir a escolha do outro participante na tentativa anterior.
}

selecionou os arranjos de contingência em grupos verbais e não-verbais, demonstrando a possibilidade de CCEs sem a necessidade da verbalização entre os jogadores. Ressalta-se ainda, que, nos grupos não verbais a organização de CCEs demorou um tempo maior para ocorrer do que nos grupos verbais. Nogueira (2009), em nova replicação, acrescentou ao estudo a possibilidade de seleção de diferentes arranjos de respostas entre os membros de grupos verbais e não-verbais, encontrando a seleção cultural dos quatro arranjos possíveis em grupos verbais e não-verbais.

A tragédia dos comuns é considerada uma das primeiras situações a serem esquematizadas pelos matemáticos interessados em dilemas sociais. $\mathrm{O}$ conceito presente na tragédia dos comuns é que em uma situação em que há um recurso renovável comum a um grupo, o grupo tende a fazer uma utilização sem restrições, resultando em um rápido esgotamento desses recursos (Dietz et al., 2001; Fiani, 2004, Hardin, 1968). A tragédia dos comuns foi inicialmente esquematizada por Lloyd (1833), um matemático que observou e relatou o mau uso de um pasto de ovelhas comuns, resultando na destruição do pasto. Assim, quanto maior o número de ovelhas alocadas no pasto, maior ganho individual para os pastores, e ainda, menor sobrevida do pasto. Assim, há o dilema entre o ganho individual em curto prazo e o ganho comum para todos os membros do grupo, em longo prazo. Se os pastores alocassem menos ovelhas no pasto comum, este poderia ser mais preservado ao longo do tempo, ocasionando um maior ganho para o grupo de pastores em longo prazo (Dietz et al., 2001)

O fenômeno da tragédia dos comuns pode ser relacionado ao conceito de macrocontingência (Glenn, 2004; Glenn \& Mallot, 2004; Mallot \& Glenn (2006), por ser possível perceber que os efeitos das respostas dos diversos organismos presentes na prática cultural (e.g. a alocação de gados no pasto comum) resultarem em um efeito cumulativo. (e.g. o fim do pasto). Esse efeito cumulativo, no entanto, não possui relação de seleção com os comportamentos observados.

A partir de elementos da vida cotidiana, envolvendo o fenômeno da tragédia dos comuns, foi esquematizado o jogo denominado Dilema dos Comuns (denominado também de Jogo dos Bens Públicos, do inglês Public Good Games - PGG), sendo baseado em situações em que há um recurso comum para o qual várias pessoas podem ter acesso. É importante ressaltar que o recurso pode ser reajustado e este reajuste é dependente das escolhas dos membros do grupo. O jogo é comparado ao uso que as pessoas fazem dos recursos renováveis da natureza, como os peixes de um ecossistema (Dietz et al., 2001). Neste caso há um grupo de pescadores em um ecossistema, com uma determinada quantidade de peixes pescados pelo grupo. A "taxa de reprodução dos peixes", isto é, o cálculo feito a partir do restante de peixes no ecossistema, após as pescarias, envolve a multiplicação por dois ou três - no último caso, resultará em uma diminuição do número de peixes para o grupo, podendo alcançar sua extinção. Hardin (1968), em um estudo acerca do fenômeno da tragédia dos comuns, reflete sobre 
os comportamentos dos organismos envolvidos em um grupo. A cada peixe retirado do tanque há um lucro na função (+1) para o pescador, já que o peixe retirado do grupo é agora uma propriedade individual e uma despesa de (-1) que é dividida entre os membros do grupo. Assim, do ponto de vista de um pescador a despesa ao ser dividida pelo grupo torna o seu lucro maior. Entretanto, se outros pescadores seguirem esta estratégia ocorrerá o fenômeno tragédia dos comuns, a extinção dos recursos. Hardin busca evitar que aconteça o fenômeno, questionando como legislar o comedimento que deve ocorrer entre os participantes do grupo, a fim de evitar o esgotamento dos recursos. Uma das opções apresentadas seria a privatização do bem comum, embora possa não parecer uma solução justa para todos que utilizem tal recurso. Essa privatização poderia ocorrer pela divisão igual do recurso entre as pessoas ou pela escolha de um indivíduo que iria administrar os recursos. Outra solução apontada por Hardin é a de que não é necessário proibir "o consumo exacerbado", mas torná-lo mais caro aos seus consumidores, o que ocorre com as multas de trânsito - o estacionamento está livre, mas estacionar naquele local acarretará em multas. Ademais, é reconhecido que as multas não solucionariam todos os problemas ocasionados pelo consumo dos bens comuns. Portanto, Hardin (1968) considera que é preciso haver um controle social para haver uma diminuição dos gastos individuais, diante de um bem comum, mas pondera sobre a supervisão dos agentes fiscalizadores quando se tratar de um bem comum de toda uma sociedade, afirmando de modo pessimista que não haveria uma solução técnica para o problema.

Jones e Rachlin (2009), Abele \& Ehrhart (2005) e De Alencar (2008) investigaram, por meio de delineamento de grupos, conceitos como o desconto do atraso e o autocontrole, além do tempo que os recursos seriam utilizados. Os resultados mostram que os jogadores tendem a consumir os bens públicos individualmente, com um baixo percentual de doações ao grupo, apesar do reajuste, que é a renovação dos recursos após cada tentativa dos participantes. O tamanho do recurso, controle social via regras de persuasão moral, o reforçamento, a punição, e a privatização dos recursos foram investigados por Brechner (1977) e Martichuski \& Bell (1991) como variáveis que controlassem o comportamento dos participantes de doar bens ao grupo e não somente consumi-los.

Brechner (1977) estudou uma situação de PGG em grupos investigando o tamanho do recurso como variável para o não esgotamento dos recursos, sendo o reajuste controlado por um esquema de reforçamento DRL. A taxa do esquema dependia da quantidade de recursos existentes, sendo um valor baixo para uma quantidade grande de recursos e vice-versa, de modo que quanto maior a quantidade de recursos restantes antes do reajuste, mais rápida a taxa de crescimento. O autor dividiu grupos com diferentes quantidades iniciais de recurso, encontrando que o grupo com menor quantidade de recurso, se utilizou do mesmo de forma mais otimizada que os outros.
Martichuski e Bell (1991) investigaram controle social, privatização, reforçamento e punição individual como variáveis independentes na situação no jogo Dilema dos Comuns. Para tanto, destinaram 15 recursos a diferentes grupos com três participantes cada com uma taxa de reajuste dependente da quantidade de recursos existentes. Os grupos eram denominados Persuasão Moral, Estrutura Básica e Privatização. O primeiro grupo recebia regras de como usar os recursos, enquanto o de Estrutura Básica não recebia regra nenhuma. O grupo Privatização tinha os recursos divididos igualmente entre os participantes desde o início do jogo, de modo que cada um recebeu cinco. Os três grupos passaram pelas condições de reforçamento, punição e extinção, em que recebiam feedbacks acerca de sua utilização dos recursos. As autoras encontraram que os grupos Persuasão Moral e Privatização fizeram um melhor uso dos recursos, principalmente nas condições de reforçamento e punição.

Dentre os estudos apresentados, Martichuski e Bell (1991) demonstraram experimentalmente que uma variável importante no Dilema dos Comuns para o não esgotamento dos recursos é o controle social, bem como Hardin (1968) já havia apontado. Borba (2014) também utilizou o controle social como variável para estudo de comportamento de pessoas em grupo em um análogo experimental de uma macrocontingência. Assim, uma vez comparado o fenômeno visto no Dilema dos Comuns com uma macrocontingência, questiona-se se o controle social também pode ser uma variável em uma situação experimental com o Dilema dos comuns simulando uma macrocontingência e, se esse controle social pode promover o entrelaçamento de contingências.

O objetivo do presente estudo é analisar o fenômeno da macrocontingência utilizando-se do jogo Dilema dos Comuns. O PGG possibilita a criação de um análogo experimental de uma macrocontingência, envolvendo um problema social voltado para a extinção de recursos públicos destinados a um determinado grupo. Entre os objetivos específicos estão: investigar o efeito: (1) das escolhas entre os participantes e (2) da comunicação entre os membros do grupo, analisando a possibilidade de resultar na produção de contingências comportamentais entrelaçadas (CCEs), um dos elementos da metacontingência, promovendo assim, uma mudança cultural no arranjo do grupo e o não esgotamento dos recursos. Assim, a interação social entre os participantes será implementada, o que poderá resultar na seleção de determinados arranjos de escolhas adotados pelos membros de um grupo, e ainda, na manutenção de tais estratégias mesmo após a suspensão do controle social.

\section{Participantes}

\section{MÉTODO}

Doze estudantes de graduação da Universidade de Brasília, cinco homens e sete mulheres, com idade entre 19 e 28 anos, participaram da pesquisa.

\section{Setting e Instrumento}

Foi utilizado um microcomputador com uma planilha desenvolvida no Microsoft Office Excel® para o 
controle de registros dos dados, recursos do grupo e escolhas dos participantes. Foram utilizados também mini cartões com os números 2, 4 e 6 com as cores amarelo, vermelho e verde, respectivamente, colocados em uma caixa para escolha dos participantes. Duas divisórias de folhas de papel cartão formaram três cabines que separaram os participantes durante todo o experimento, impedindo contato visual e acesso às escolhas dos membros do grupo. A Figura 2 mostra a disposição da experimentadora, dos participantes, e ainda, os instrumentos e materiais utilizados.

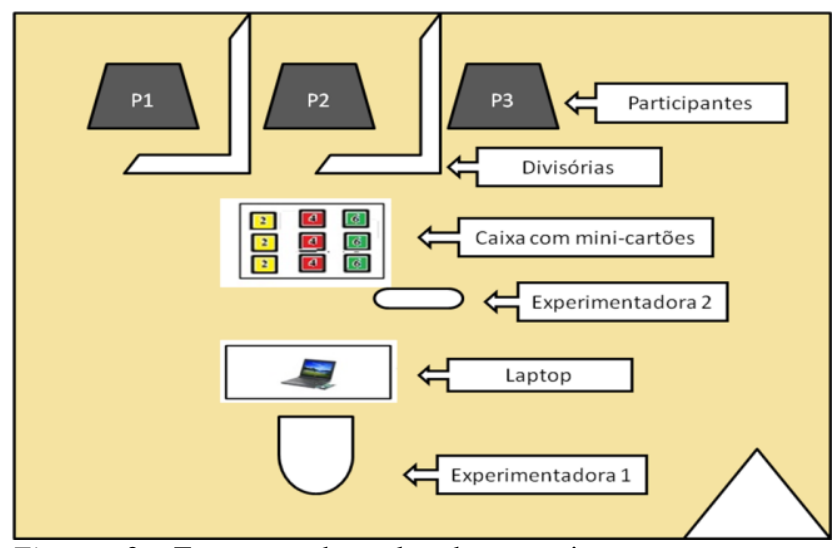

Figura 2. Esquema da sala de experimentação com as experimentadoras e materiais utilizados.

\section{Procedimento}

O experimento consistiu da aplicação do Jogo Dilema dos Comuns em três condições: linha de Base (Condição A) e duas condições de intervenção com o controle social, a Condição B1, em que os participantes tiveram acesso a escolha dos colegas e a B2, em que foi possibilitado o diálogo entre eles.

A seguinte instrução foi apresentada aos participantes no início do experimento:

Olá! Imaginem que vocês fazem parte de um grupo. Esse grupo possui um tanque com 100 peixes. Cada um dos participantes do grupo terá a opção de retirar peixes do tanque escolhendo um dos mini cartões da caixa ao lado. Quando todos escolherem seus mini cartões, ou seja, for retirada determinada quantidade de peixes, os peixes restantes no tanque do grupo vão se reproduzir e haverá um novo montante no tanque. Após a reprodução, eu vou anunciar quantos peixes possuem no tanque. Ao fim de cada condição do jogo, os recursos restantes serão divididos entre vocês. Os peixes serão trocados por uma quantia de $R \$ 0,03$ ao fim do jogo. Durante essa condição vocês não deverão se ver, conversar ou ter acesso à escolha dos outros participantes do grupo. Cabe a vocês fazerem uso desses peixes da maneira como acharem melhor.

Foram três grupos experimentais com alteração da ordem das condições experimentais e um grupo controle, que foram assim denominados: GE1, GE2 e GE3 e GC. As sessões duraram em média 50 minutos. Na
Tabela 1 estão descritos os grupos e os delineamentos programados para todos os grupos citados anteriormente.

Tabela 1

Descrição dos Grupos com os Delineamentos Experimentais Correspondentes.

\begin{tabular}{cc}
\hline Grupos & Delineamentos \\
\hline GE1 & A B1 B2 A \\
GE2 & A B2 B1 A \\
GE3 & A B1 B1 A \\
GC & A A A A \\
\hline
\end{tabular}

No início da sessão, os participantes foram instruídos e apresentados a uma situação em que possuíam um tanque com 100 peixes e poderiam pescar para si mesmos por meio de mini cartões inseridos em uma caixa. Os peixes restantes no tanque iriam se reproduzir e a quantidade de peixes após a reprodução era comunicada a todo o grupo. Os peixes restantes ao fim de uma condição seriam divididos igualmente entre os participantes e cada peixe equivaleria a um ponto. Os participantes deveriam retirar um mini cartão amarelo, vermelho ou verde da caixa, correspondentes a 2, 4 e 6 peixes, respectivamente. Após a escolha dos três participantes, havia um reajuste no número de peixes, dependendo do montante retirado do recurso inicial. Assim, uma quantidade menor de recursos retirados pelo grupo resultava em um aumento destes a cada tentativa, enquanto uma maior retirada dos recursos pelo grupo resultava em sua diminuição. Na Tabela 2 estão dispostos os valores do montante (total retirado pelo grupo) e do reajuste em porcentagem.

Tabela 2

Reajuste e Restante dos Recursos na Primeira Tentativa, de Acordo com o Montante Retirado pelo Grupo.

\begin{tabular}{ccc}
$\begin{array}{c}\text { Montante } \\
\text { retirado pelo } \\
\text { grupo }\end{array}$ & $\begin{array}{c}\text { Reajuste } \\
(\%)\end{array}$ & $\begin{array}{c}\text { Recursos restantes da } \\
\text { primeira tentativa após } \\
\text { reajuste }\end{array}$ \\
\hline 6 & 9 & 102,46 \\
8 & 9 & 100,28 \\
10 & 8,5 & 97,65 \\
12 & 8,5 & 95,48 \\
14 & 8,0 & 92,88 \\
16 & 8,0 & 90,72 \\
18 & 8,0 & 88,56 \\
\hline
\end{tabular}

De acordo com o reajuste mostrado na Tabela 2, para que os peixes não se esgotassem, a escolha dos participantes deveria ser pelo cartão amarelo ( 2 peixes). Ainda assim, se os recursos já estivessem em uma quantidade abaixo de 70 peixes, eles se esgotariam. Caso dois participantes fizessem a escolha pelo cartão amarelo e um deles pelo vermelho desde o início, com o tanque do grupo possuindo no mínimo 97 peixes, os peixes também aumentariam de número. $\mathrm{O}$ critério de finalização das sessões foi determinado pela quantidade de peixes no tanque $-\leq$ a 18 ou 200 peixes ou, ainda, pela oscilação entre consumo irrestrito e otimizado dos recursos com quantidade entre 150 a 200, ou seja, caso os participantes alternassem suas escolhas entre retirar muitos peixes e 
retirar poucos peixes, resultando na oscilação dos recursos. A seguir serão descritas as condições experimentais.

Linha de Base - Na Condição A, o objetivo foi realizar um análogo experimental de uma macrocontingência. Nesta condição, os participantes foram instruídos a não manter contato visual, interações verbais como tampouco tinham acesso às jogadas dos demais participantes, impedindo possíveis combinações e forçando-os a jogarem individualmente. As escolhas eram feitas no momento em que a experimentadora autorizava e aconteciam simultaneamente.

Condições B1 e B2 - A interação e o controle social foram implementadas pela permissão de interações sociais no jogo Dilema dos Comuns. Nestas condições, o jogo era o mesmo da Linha de Base, mas as escolhas dos participantes eram feitas sequencialmente e eram socializadas pela experimentadora, que repetia em voz alta a escolha de cada participante. Na Condição B1, foi permitido aos participantes terem acesso às escolhas dos demais membros do grupo, por meio a das escolhas de cada participante. Na Condição B2, além disso, foi permitido o diálogo entre os participantes, sendo possível a combinação de estratégias em grupo. Esse diálogo foi permitido a cada duas tentativas de escolhas do grupo, sendo considerada uma tentativa todos os participantes escolherem seus cartões. O tempo destinado ao diálogo entre os participantes era de um minuto e ao final dele, ocorriam novas escolhas dos participantes.

Dessa forma, as variáveis independentes desse estudo foram as descrições da experimentadora na condição B1 e o contato social entre os participantes na condição B2. As condições B2 tiveram em média 30 tentativas, enquanto a condições B1 tiveram, em média, 20 tentativas, com exceção do GE2, em que houve 39 tentativas. A Tabela 3 mostra a organização das condições e das variáveis sociais introduzidas.

Tabela 3

Descrição das Condições A, B1 e B2, de Acordo com as Variáveis Sociais Introduzidas em Cada Condição.

Condições Variáveis sociais

\begin{tabular}{|c|c|c|c|}
\hline & Se ver & Se falar & $\begin{array}{c}\text { Saber a escolha } \\
\text { do outro }\end{array}$ \\
\hline \multicolumn{4}{|l|}{ A - Linha de Base } \\
\hline $\begin{array}{l}\text { B1 - Não visual e } \\
\text { não verbal }\end{array}$ & & & $\mathrm{X}$ \\
\hline $\begin{array}{c}\text { B2 - Não visual e } \\
\text { verbal }\end{array}$ & & $X$ & $X$ \\
\hline
\end{tabular}

Os participantes receberam a mesma instrução da Condição de Linha de Base, alterando apenas as instruções específicas referentes ao acesso à jogada do outro participante em B1, e ainda, que poderiam conversar entre si em B2, que foram adicionadas ao fim da instrução geral, no início de cada condição.

Instrução Condição B1: "Nessa condição a escolha será seqüencial e eu irei dizer a escolha de cada um em voz alta. Dessa forma, vocês terão acesso ao que os outros estão escolhendo.
Instrução Condição B2: Nessa condição vocês poderão se comunicar em alguns momentos. A comunicação deve ser realizada apenas quando solicitada.

\section{RESULTADOS}

As Figuras 3, 4 e 5 apresentam os dados obtidos de todos os grupos (GE1, GE2, GE3 e GC), em suas respectivas condições. A sequência da exposição dos grupos às condições experimentais está descrita nas legendas. A Figura 3 apresenta dados relativos ao recurso do grupo - com aumento ou esgotamento do bem público. As Figuras 4 e 5, por sua vez, apresentam as escolhas feitas por cada membro do grupo.

A Figura 3 traz a disposição dos recursos nas condições experimentais, sendo representado o aumento ou a diminuição dos recursos em cada condição, nos quatro grupos. Todos os grupos passaram por uma exposição à Linha de Base (LB) antes e após a exposição às condições experimentais B1 e B2. Houve esgotamento dos recursos nas sessões iniciais de Linha de Base em todos os grupos, o que não aconteceu nas sessões de retorno à Linha de Base, com exceção do grupo controle, em que houve esgotamento dos recursos em todas as condições. Nos três grupos experimentais houve aumento dos recursos em duas condições e consumo de todos os recursos nas demais. Em GE1 e GE2, o aumento dos recursos ocorreu na condição verbal (B2) e no retorno à linha de base (LB2), enquanto em GE3 ocorreu na segunda exposição à condição não verbal (B1.2). No GE1, em que os participantes foram expostos inicialmente à condição não verbal e, posteriormente à condição verbal, observa-se que houve esgotamento dos recursos na condição não verbal, não ocorrendo tal resultado na condição verbal. Além disso, é interessante observar que nesse retorno à $\mathrm{LB}$, os participantes alternaram suas jogadas entre consumo de mais e de menos recursos, quando próximos a 200 peixes no tanque. No GE2, em que o grupo foi exposto inicialmente à condição verbal, percebe-se que não houve esgotamento do recurso, entretanto, tal esgotamento voltou a ocorrer na condição não verbal, em que os participantes apenas sabiam a escolha do outro jogador. Em GE3, em que houve duas exposições à condição não verbal, percebe-se que o esgotamento dos recursos ocorreu somente na primeira exposição.

A Figura 4 mostra a porcentagem das escolhas do cartão amarelo pelos participantes nas diferentes condições, seguindo a sequência de exposição. Os participantes, em geral, apresentaram baixas porcentagens de escolha pelo cartão amarelo na primeira exposição à LB. Somente GE2 mostrou uma porcentagem de $58 \%$ das escolhas do cartão amarelo. Entretanto, como essas escolhas não foram coordenadas, não produziu aumento do recurso. Foram denominadas escolhas coordenadas as escolhas combinadas entre os participantes ou que seguiram um padrão de escolhas por pelo menos três tentativas seguidas. 

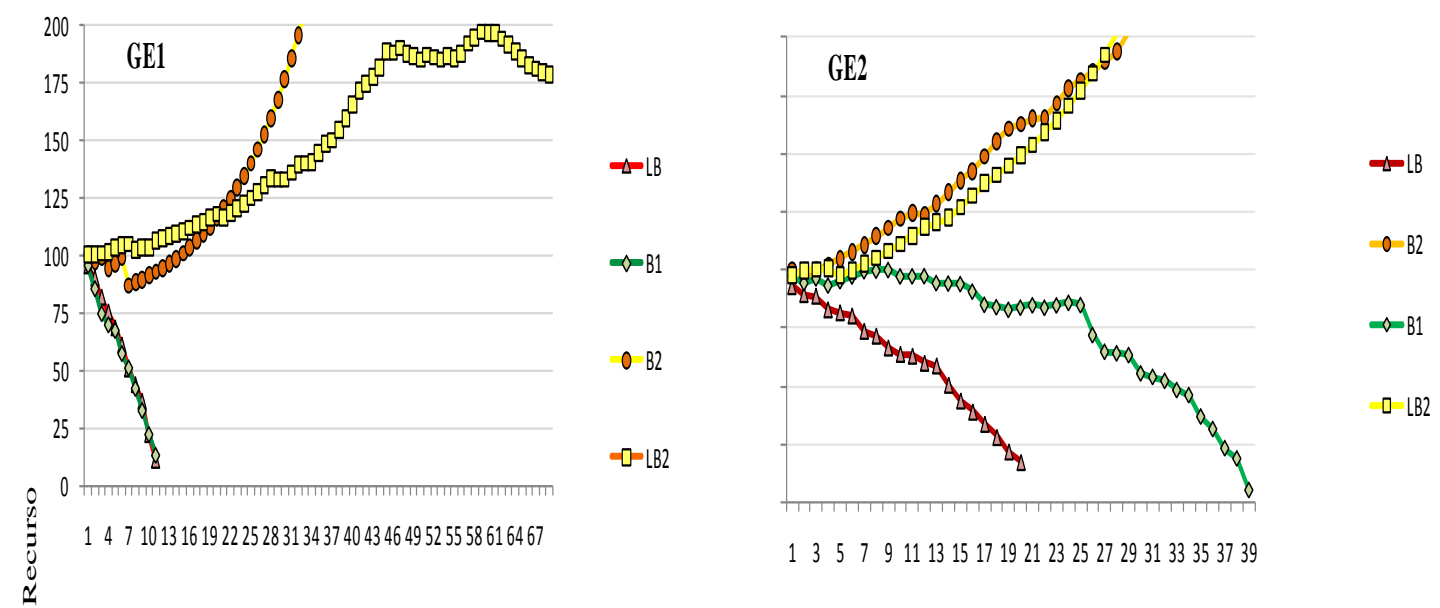

13579111315171921232527293133353739
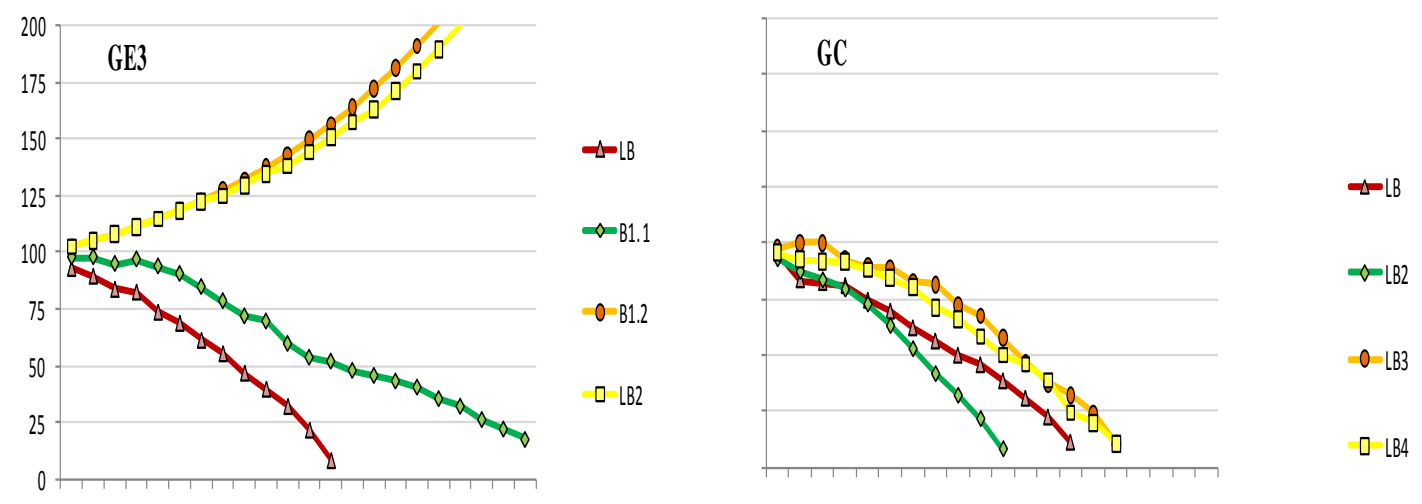

12345678910111213141516171819202122

1234567891011121314151617181920

Tentativas

Figura 3. Quantidade de recursos ao longo das tentativas em todas as condições da pesquisa. Na parte superior encontram-se os grupos experimentais GE1 e GE2 e na parte inferior o grupo experimental GE3 e o grupo controle GC.

Em todos os grupos houve aumento das escolhas pelo cartão amarelo nas condições em que seus recursos aumentaram. No GE1, na Condição B1.1 não se verificou aumento considerável das escolhas do cartão amarelo, apesar dos participantes terem acesso à escolha dos demais. Na condição verbal (B2), entretanto, verificou-se um aumento das escolhas desse cartão pelo grupo para $90 \%$. No retorno à LB, observase uma continuidade da escolha do cartão amarelo por dois participantes. Um dos participantes escolheu o cartão vermelho, o que pode ser constatado na Figura 5 na escolha coordenada Amarelo/Amarelo/Vermelho (AAV) que produziu o aumento do recurso.

No GE2, inicialmente exposto à condição verbal, observa-se um aumento das escolhas do cartão amarelo pelo grupo, chegando a uma porcentagem de mais de $75 \%$. Entretanto, houve uma diminuição dessas escolhas na condição não verbal, e consequente esgotamento dos recursos (ver Figura 3). No retorno à Linha de Base, no entanto, a escolha do cartão amarelo nesse grupo voltou a aumentar, chegando aos $100 \%$ para um dos participantes e, novamente a mais de $75 \%$ para o grupo. No GE3, exposto a duas condições não verbal consecutivas, a escolha do cartão amarelo pelo grupo aumentou já na primeira exposição, entretanto, isso foi devido principalmente à escolha de um participante, o que não foi suficiente para haver o não esgotamento dos recursos. Na segunda exposição à condição não verbal, essas escolhas atingiram 100\% das escolhas dos participantes. No retorno à LB, a escolha desse cartão por um participante diminuiu, mas a escolha do grupo permaneceu próxima a 100. Na Figura 5 , pode-se perceber também que esse participante (P2) escolheu o cartão vermelho. No grupo controle, percebe-se uma tendência por escolher mais amarelos do P2, entretanto, essa escolha diminuiu ao longo das exposições e porque não foi acompanhada pelos demais participantes determinou o esgotamento dos recursos. A escolha do grupo controle pelo cartão amarelo foi sempre menor que $50 \%$ em todas as condições. 

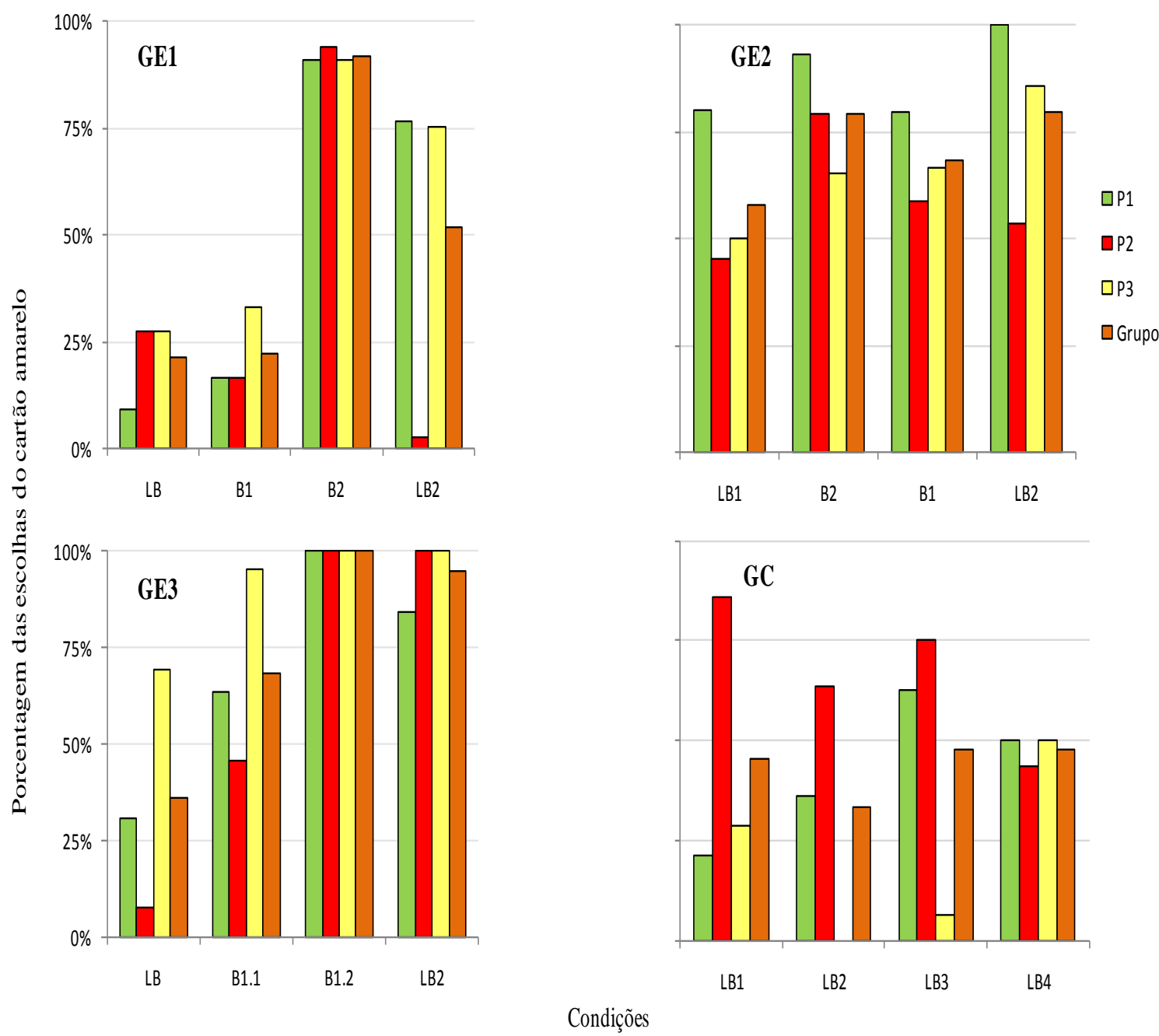

Figura 4. Porcentagem das escolhas do cartão amarelo dos participantes, por grupo, durante as condições LB, B1 e B2. Na parte superior encontram-se os grupos GE1 e GE2 e na parte inferior o GE3 e o GC.

A Figura 5 mostra as escolhas coordenadas AAA e AAV, que representam, respectivamente, as escolhas do cartão amarelo e de dois cartões amarelos e um vermelho por todos os participantes de um grupo. Os entrelaçamentos ou as escolhas coordenadas AAA e AAV produziam aumento dos recursos dependendo da quantidade de recursos existentes. No caso da escolha AAA, era necessário que os recursos estivessem na faixa dos 70 peixes, enquanto a escolha coordenada AAV somente resultaria em um aumento dos recursos caso fosse realizada desde o início, com no mínimo 97 peixes no tanque (recursos do grupo). No entanto, tais entrelaçamentos AAA e AAV podem também resultar em menor ganho individual de peixes. Esses entrelaçamentos ocorreram quando a escolha do cartão amarelo influenciava a escolha de outro participante ou, ainda, por meio da combinação de estratégias, resultando em baixas quantidades de retirada do tanque, o que produziu aumento do recurso (conseqüência cultural externa às coordenações ou às CCEs e ao produto agregado). Dessa forma, é possível perceber que tais entrelaçamentos só podem ocorrer nas condições B1 e B2, em que há a possibilidade da influência da escolha do outro e da combinação de estratégias. Como pode ser observado na Figura 7, os grupos GE1, GE2 e GE3 mostram a presença das escolhas coordenadas entre AAA e AAV nas condições em que houve controle social. Entretanto, GE1 apresentou escolhas individuais alternadas entre retirar 2 e 6 peixes em LB2, produzindo aumento e esgotamento dos recursos. Finalmente, todas as escolhas coordenadas dos grupos GE1, GE2 e GE3 foram seguidas pelo aumento do recurso nestas condições.

No GE1, observa-se que não houve escolhas coordenadas entre os cartões AAA ou entre os cartões AAV em LB1. Na condição não verbal (B1), mesmo conhecendo as escolhas dos demais participantes, não houve coordenação AAA entre as escolhas dos participantes, e ainda, uma baixa porcentagem de escolhas entre os cartões amarelo com um vermelho (AAV $8,33 \%$ ). Na condição verbal (B2), as escolhas coordenadas entre cartões amarelos (AAA) e dois cartões amarelos com um vermelho (AAV) alcançaram mais de $90 \%$ das escolhas, em todas as tentativas. Finalmente, em LB2, houve uma queda dos entrelaçamentos com o cartão AAA 
e aumento de escolhas coordenadas AAV (47\%). Entretanto, como essas escolhas foram feitas desde o início dessa condição, produziram aumento dos recursos. E ainda, em duas ocorrências, observou-se a diminuição dos recursos quando a quantidade de peixes aproximou-se de sua capacidade máxima - 200 peixes no tanque. Naquelas tentativas, poderia haver consumo sem prejuízo ao grupo, visto que as escolhas coordenadas no início da condição produziram um número de peixes que tornava possível alternar o consumo maior e menor dos recursos sem que os recursos chegassem a um número mínimo. Esses consumos ocorreram com o cartão verde, o que explica a baixa porcentagem das escolhas coordenadas entre amarelo e vermelho (AAV), apesar do não esgotamento dos recursos.

No GE2, observa-se uma alta porcentagem de escolhas de cartões AAV desde LB1, no entanto, essas escolhas não resultaram em aumento dos recursos, pois ocorreram quando os recursos já estavam abaixo do limiar, o que impossibilitava aumento. As porcentagens de escolhas de cartão amarelo AAA em LB1 foram abaixo de $25 \%$. Na condição verbal (B2), houve um aumento dos entrelaçamentos de AAA para 50\% das escolhas, atingindo os $75 \%$ das escolhas o total de AAA e AAV, o que resultou no aumento dos recursos. Esses entrelaçamentos voltaram a diminuir na condição não verbal (B1), tendo AAA uma porcentagem de ocorrência de $30 \%$ e o total de AAA e AAV de $58 \%$. Essas escolhas não foram coordenadas de forma a resultar em aumento dos recursos. No retorno à linha de base (LB2), não obstante, houve um novo aumento das escolhas desses dois conjuntos de cartões (AAA e AAV), atingindo um total de $90 \%$ das escolhas, o que conduziu ao aumento dos peixes no tanque.
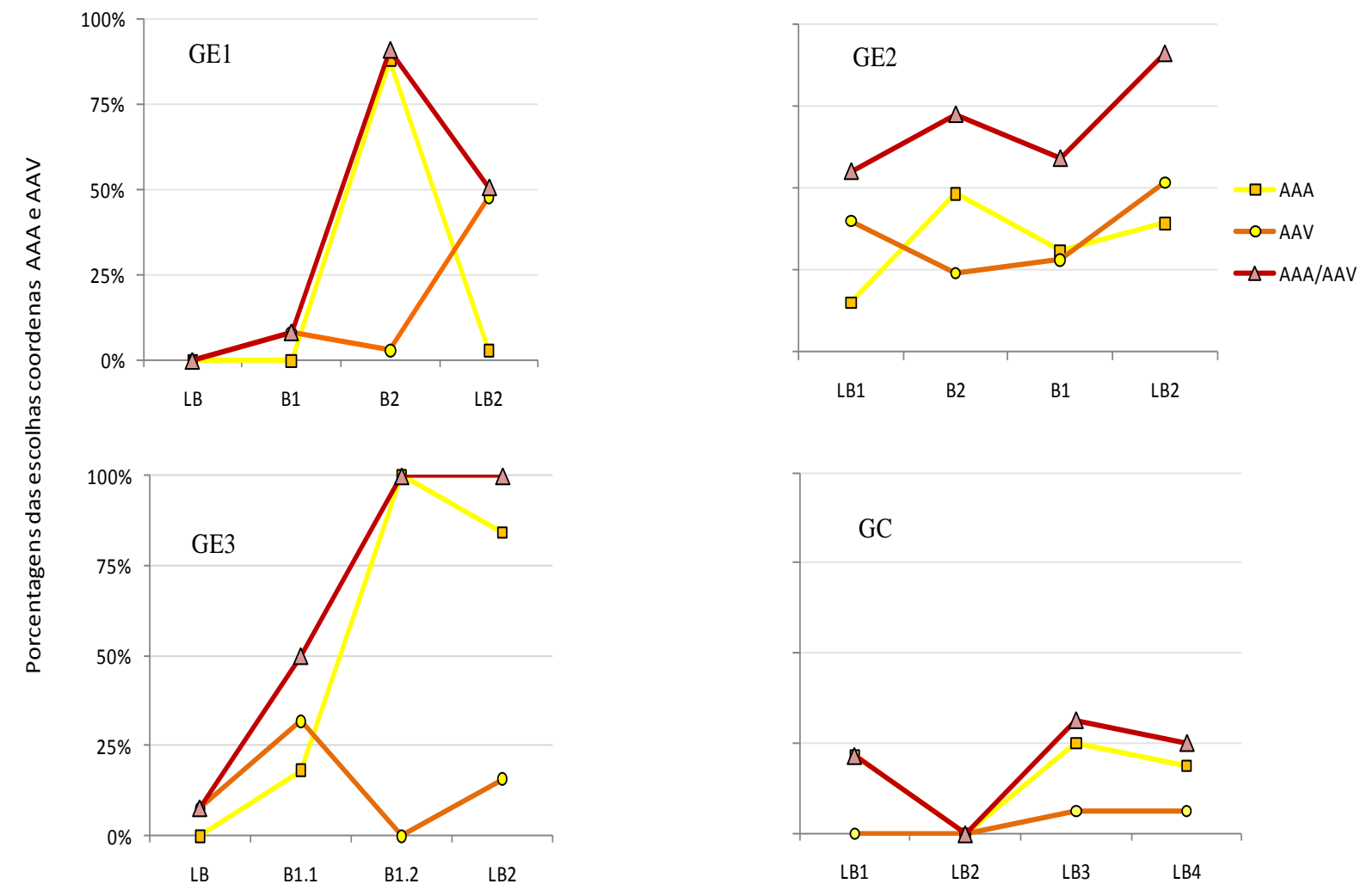

Condições

Figura 5. Porcentagem das escolhas coordenadas de cartões amarelos (AAA) e dois cartões amarelos com um vermelho (AAV) dos participantes dos grupos GE1, GE2, GE3 e GC, nas condições experimentais. Na parte superior encontram-se os grupos experimentais 1 e 2 e na parte inferior o grupo experimental 3 e o grupo controle.

O GE3 mostrou baixa porcentagem de escolhas do cartão AAA em LB1. Na primeira exposição à condição não-verbal (B1.1), houve um aumento das escolhas coordenadas amarelas, principalmente das escolhas com AAV, sendo o total (AAA e AAV) dessas escolhas 50\%. No entanto, essas escolhas não resultaram em aumento dos recursos. Em B1.2, as escolhas coordenadas do cartão AAA representaram a totalidade das escolhas dos participantes. E, em LB2, a soma das escolhas AAA e AAV representou a 
totalidade das escolhas, havendo uma pequena queda na percentagem de escolhas por cartões AAA.

O Grupo Controle (GC) mostrou uma baixa percentagem de escolhas de cartões AAA em todas as exposições, tendo as escolhas totais AAA e AAV atingido a mais alta porcentagem (31\%) em LB3. Entretanto, essas escolhas não foram suficientes para resultar em aumento dos recursos.

\section{DISCUSSÃO}

O experimento desenvolvido a partir do jogo Dilema dos Comuns mostrou-se um meio efetivo para a análise dos elementos componentes nos conceitos de macrocontingência e metacontingência propostos por Glenn (2004), Glenn e Mallot (2004) e Mallot e Glenn (2006). Na primeira condição de linha de base (A), houve um maior consumo de peixes, sugerindo que as escolhas foram emitidas com base no maior ganho individual. Este padrão de respostas pode ser interpretado por meio do conceito de macrocontingência (Glenn, 2004; Mallot \& Glenn, 2004), segundo o qual um conjunto de contingências tríplices produz um efeito cumulativo no meio social (Mallot \& Glenn, 2006). Dessa forma, observa-se a produção de um macrocomportamento na primeira condição de linha de base, sendo a redução do número de peixes do tanque o efeito cumulativo. (Glenn, 2016).

A ausência de relação funcional entre as escolhas nas primeiras condições de Linha de base faz com que essa condição possa ser analisada como um análogo experimental de macrocontingência. A Figura 6 mostra a extinção dos peixes do grupo relacionada ao conceito de macrocontingência:

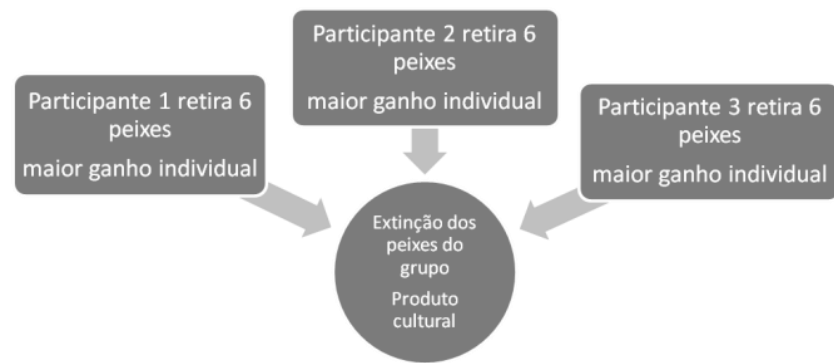

Figura 6. Esquema a partir dos dados obtidos, nas condições em que não foi observada a seleção cultural - macrocontingências (Glenn, 2004; Mallot \& Glenn, 2004). Scheme from data obtained under conditions that did not observe the cultural selection - macrocontingências (Glenn, 2004; Mallot \& Glenn, 2004).

No transcorrer das condições, novas relações foram observadas, ilustrando elementos componentes do conceito de metacontingência a exemplo do aumento das escolhas coordenadas AAA e AAV, nas Condições B2. Em B2, era permitido aos participantes conversarem em alguns momentos pré-determinados do jogo, o que facilitou a coordenação das escolhas. $\mathrm{O}$ comportamento verbal dos participantes ilustra o controle social estabelecido nessa condição. A Tabela 4 mostra alguns exemplos das declarações verbais dos participantes:

Nos grupos GE1 e GE2 o comportamento verbal foi necessário para produzir o entrelaçamento das contingências, pois na condição B1 não houve coordenação AAA e AAV, as quais produziam aumento dos peixes. No GE2, o grupo foi exposto inicialmente à Condição B2, havendo seleção cultural, entretanto, na Condição B1, essa seleção não ocorreu, havendo novamente escolhas que ocorriam de modo individual (como sugerido pelas Figuras 4 e 5). Dessa forma, como bem afirmaram Skinner (1953) e Glenn (1991) acerca do papel do comportamento verbal no entrelaçamento de contingências comportamentais, ele serve como o suporte das contingências não verbais, sendo o facilitador da coordenação entre essas contingências.

Tabela 4

Exemplos de Declarações Verbais dos Participantes dos Grupos Experimentais na Condição Verbal.

Grupo Exemplos de falas dos participantes

G1 "Eu acho que se todo mundo escolher o amarelo, os peixes se reproduzem mais.

G1 "É pra escolher o cartão amarelo, pra aumentar os peixes"

G1 "Lembra que o que sobra dos peixes no fim do jogo é dividido por todo mundo? Então, cara, escolhe o amarelo!"

G2 "E se todo mundo colocar a mesma cor?"

G2 "Todo mundo tem que pôr amarelo, é isso!"

O GE3 foi programado após a obtenção dos dados dos grupos GE1 e GE2, com o objetivo de investigar $\mathrm{o}$ surgimento de entrelaçamento das contingências na ausência de interações verbais. Esse grupo possibilitou a comparação com os dados de Costa (2009) e Nogueira (2009). Consistente com os dados obtidos nessas pesquisas, o entrelaçamento das escolhas ocorreu de forma mais lenta, apenas após a segunda exposição à Condição B1.

Na condição B2 dos Grupos GE1 e GE2 e na segunda Condição B1 no GE3 houve seleção cultural, sendo possível relacionar os dados obtidos ao conceito de metacontingência (Mallot \& Glenn, 2006). Nesse caso, as CCEs seriam as escolhas coordenadas dos cartões amarelos AAA, produzindo um total de seis peixes retirados do tanque - o produto cultural. De acordo com o conceito, a seleção cultural ocorre por meio da consequência cultural, isto é, do aumento e sustentabilidade dos peixes, nas condições em que foi observado o entrelaçamento. O esquema é apresentado na Figura 7. 


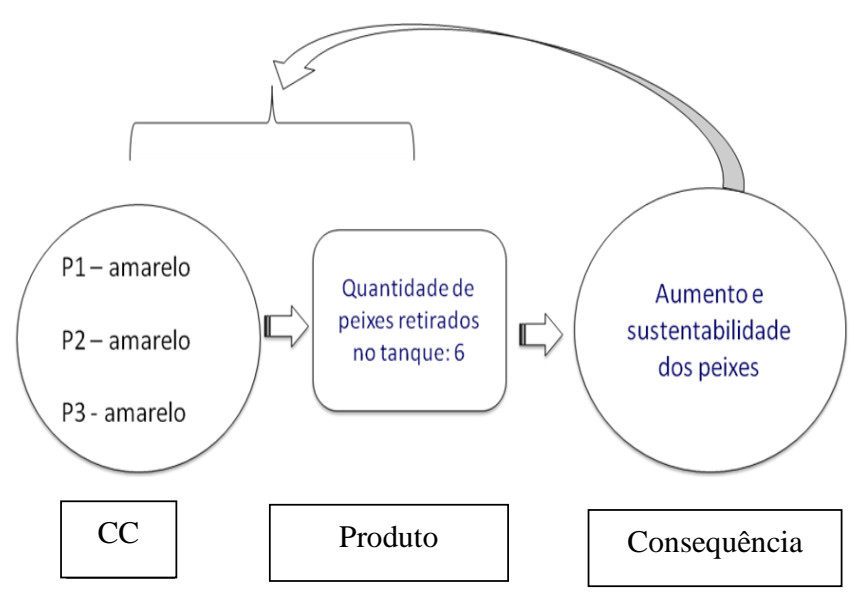

Figura 7. Esquema dos dados obtidos - conceito de metacontingência (Mallot \& Glenn, 2006), representando os elementos de contingências comportamentais entrelaçadas (CCEs), produto agregado e consequiência cultural externa a este arranjo

A consequência cultural foi o aumento e a sustentabilidade dos peixes, devido a um reajuste do número de peixes. Nas primeiras condições de Linha de Base e no grupo controle, em que não houve entrelaçamento, o reajuste não apresentou função de consequência cultural, não existindo seleção cultural.

O presente estudo mostrou uma seleção cultural com a consequência cultural apresentada em esquema de reforçamento contínuo. É interessante ressaltar que futuras pesquisas devem avaliar a inserção de um esquema intermitente de reforçamento no Dilema dos Comuns, investigando as relações com a seleção e intervenção cultural. Estudos recentes mostraram ser possível a seleção cultural com o esquema de reforçamento variável (VR2) por meio de um programa de computador que permite a interação de comportamentos dos participantes (Amorim, 2010) ou ainda, por meio do jogo Dilema dos Prisioneiros (Costa, 2009; Nogueira, 2009; Ortu e Cols., 2008).

\section{REFERÊNCIAS}

Abele \& Ehrhart (2005). The Timing Effect in Public Good Games. Journal of Experimental Social Psychology, 41, 470-481.

Amorim, C. V. (2010). Análogos experimentais de metacontingências: Efeitos da intermitência da conseqüência cultural. Dissertação de Mestrado, PUC - SP, São Paulo.

Baia, F. (2008) Microssociedades no laboratório: o efeito de conseqüencias ambientais Externas sobre as contingências comportamentais entrelaçadas $e$ seus Produtos culturais. Dissertação de Mestrado. Universidade de Brasília, Brasília.

Borba, A. V., Tourinho, E.Z, (2010), Effects of Exposure to Macrocontingencies in the Production of Ethical Self-Management Responses, Anais da Conference of Association of Behavior Analysis, San Antonio, Texas, EUA.
Brechner, K. C. (1977). An experimental analysis of social traps. Journal of Experimental Social Psychology, 13, 552-564.

Brocal, A. L. (2010). Análogos experimentais de metacontingências: o efeito da retirada da conseqüência individual. Dissertação de mestrado, PUC - SP, São Paulo.

Bullerjhann, P. B. (2009). Análogos experimentais de evolução cultural: o efeito das consequências culturais. Dissertação de Mestrado. PUC-SP, São Paulo.

Caldas, R. A. (2009). Análogos experimentais de seleção e extinção de metacontingências. Dissertação de Mestrado. PUC-SP, São Paulo.

Costa, D. C. (2009). Dilema do Prisioneiro: efeito das consequências individuais e culturais sobre as escolhas de cooperação e competição. Dissertação de Mestrado. Universidade de Brasília, Brasília.

Cruz, A. T. (2009). Regras de controle tecnológico e de controle cerimonial: Efeitos sobre práticas culturais de Microssociedades experimentais. Dissertação de Mestrado. Universidade de Brasília, Brasília.

De Alencar, A. (2008) A teoria dos jogos como metodologia de investigação científica para a cooperação na perspectiva da psicologia evolucionista PSICO $\Psi$ v. 39, n. 4, pp. 522-529.

Dietz et Al. (2002). The Drama of the Commons. Em Committee on the Human Dimensions of Global Change. (Orgs) The Dramma of Commons (pp. 3-36) Washington: NATIONAL ACADEMY PRESS

Fiani, R. (2004). Teoria dos Jogos: para cursos de Administração e Economia. Rio de Janeiro: Elsevier.

Gadelha, T. C. (2010). Evolução cultural em análogos experimentais de metacontingências: seleção de diferentes produtos agregados. Dissertação de Mestrado, PUC - SP , São Paulo

Glenn, S. S. (1986/2005). Metacontingências em Walden Dois. (R. C. Martone \& D. S. C. Ferreira, Trads.) Em J. C. Todorov, R. C. Martone, M. B. Moreira (Orgs.), Metacontingências: comportamento, cultura $e$ sociedade (pp. 13-28). Santo André: ESETec.

Glenn, S. S. (1989). Verbal behavior and cultural Practices. Behavior Analysis and Social Action, 7, 1015

Glenn, S. S. (1991). Contingencies and metacontingencies: relations among behavioral, cultural, and biological evolution. Em P. A. Lamal (Orgs), Behavioral analysis of societies and cultural practices (pp. 39-73). Washington, DC: Hemisphere.

Glenn, S. S. (2004). Individual behavior, culture and social change. The Behavior Analyst, 27, 133-151.

Glenn, S. S., \& Malott, M. E. (2004). Complexity and selection: implications for organizational Change. Behavior and Social Issues, 13, 89-106.

Glenn, S. S., Malott, M. E., Andery, M.A.P.A., Benvenuti, M., Houmanfar, R.A., Sandaker, I., Todorov, J.C., Tourinho, E.Z., \& Vasconcelos, L.A. (2016). Toward consistente terminology in a behaviroist approach to cltural analysis. Behavior and Social Issues, 25, 1127. 
Hardin, G. (1968) The Tragedy of the Commons. Science, 162(1968):1243-1248.

Jones, B. A., \& Rachlin, H. (2009). Delay, probability, and social discounting in a public goods game. Journal of the Experimental Analysis of Behavior, 91, 61-74.

Leite, F. L. (2009). Efeitos de instruções e história experimental sobre a transmissão de práticas de escolha em microculturas de laboratório. Dissertação de Mestrado. UFPA, Belém.

Lima, S. P., (2005). Crime organizado e lavagem de dinheiro: uma aplicação das

teorias dos jogos e de redes neurais para reconhecimento e descrição de Padrões, Tese

de doutorado. Universidade Federal de Santa Catarina. Santa Catarina.

Malott. M. \& Glenn, S.S. (2006). Targets of intervention in cultural and behavioral change. Behavior and Social Issues, 15, 31-56

Martone, R. C. (2008). Efeito de consequências externas e de mudanças na constituição do grupo sobre a distribuição dos ganhos em uma metacontingência experimental. Tese de Doutorado. Universidade de Brasília, Brasília.

Martichuski \& Bell (1991). Reward, Punishment, Privatization and Moral Suasion in a Commons Dillema. Journal of Applied Social Psychology. 21, 16, 1356-1369.

Nogueira C. P. V. (2009). Seleção de Diferentes Culturantes no Dilema do Prisioneiro: Efeito da Interação entre a Consequência Cultural, Escolhas Simultâneas ou Sequenciais e a Comunicação. Dissertação de Mestrado, Universidade de Brasília, Brasília.

Ortu, Glenn, \& Woelz (2008). A Prisoner's Dilemma Game with an external selecting agent: $a$ metacontingency experiment. Trabalho apresentado na $34^{\mathrm{a}}$ Convenção Anual da ABA, San Diego, California.

Pereira, J. M. C. (2008). Investigação experimental de metacontingências: separação do produto agregado e da consequência individual. Dissertação de Mestrado, PUC-SP, São Paulo.

Skinner, B. F. (1953/2000). Ciência e Comportamento Humano (J. C. Todorov \& R. Azzi, trads.). São Paulo: Martins Fontes.

Skinner, B.F. (1957). Verbal Behavior New York: Appleton-Century-Croft

Skinner, B. F. (1981). Selection by consequences. Science, 213, 501-504.

Vichi C. (2004). Igualdade ou desigualdade: manipulando um análogo experimental de prática cultural em laboratório. Dissertação de Mestrado. PUC - SP, São Paulo.

Vieira, C. M. (2010). Condições antecedentes participam de metacontingências? Dissertação de mestrado, PUC - SP, São Paulo.

Yi, R., \& Rachlin, H. (2004). Contingencies of reinforcement in a five-person Prisoner's Dilemma. Journal of the Experimental Analysis of Behavior, 82, 161-176. 